\title{
PERSPECTIVE
}

\section{Data needs and models for the opioid epidemic}

\author{
Carlos Blanco $\mathbb{D}^{1 凶}$, Melanie M. Wall ${ }^{2}$ and Mark Olfson ${ }^{2}$ \\ This is a U.S. government work and not under copyright protection in the U.S.; foreign copyright protection may apply 2021
}

The evolving nature of the opioid epidemic and continued increases in overdose deaths highlight a need for fundamental change in the collection and use of surveillance data to link them to implementation of effective service, treatment, and prevention approaches. Yet at present, the quality and timeliness of US surveillance data often limits data-driven approaches. We review current information needs, summarize limitations of existing data, propose complementary surveillance resources, and provide examples of promising approaches designed to meet the needs of data end-users. We conclude that there is a need for an approach that focuses on the needs of data end-users, such as public health systems leaders, policy makers, public, nonprofit and prepaid healthcare systems, and other systems, such as the justice system. Such an approach, which may require investments in new infrastructure, should prioritize improvements in data timeliness, sample representativeness, database linkage, and increased flexibility to adapt to shifts in the environment, while preserving the privacy of survey participants. Use of simulations, distributed research and data networks, alternative data sources, such as wastewater or digital data collection and use of blockchain technology, are some of promising avenues toward an improved and more user-centered surveillance system.

Molecular Psychiatry (2022) 27:787-792; https://doi.org/10.1038/s41380-021-01356-y

The current policy discourse over federal infrastructure has reignited interest in the potential for better data and modeling methods to help address the US opioid crisis. Continued increases in overdose deaths, which appear to have accelerated during the COVID pandemic, highlight a need for fundamental change in the collection and use of surveillance data [1, 2]. For example, provisional data from the National Vital Statistics System (NVSS) estimates 90,722 overdose deaths in the 12-month period ending in November 2020, up from 70,357 in the preceding 12 months [3]. The evolving nature of the epidemic further requires linking timely surveillance data to implementation of effective service, treatment, and prevention approaches [4]. Yet at present, the quality and timeliness of US surveillance data often limits data-driven policy and intervention approaches, despite their utility in other health crises $[5,6]$. For example, rapid identification of an HIV outbreak in Scott County, Indiana, led to declaration of a public health emergency and establishment of syringe-exchange program to curtail the outbreak [7] and rapid identification of COVID-19 cases continues to be key to determining public health responses to the disease [8]. Yet similar approaches are not currently available at scale for the opioid epidemic.

In this Perspective, we focus on surveillance data needed to contain and eventually end the opioid crisis. We outline current information needs, summarize limitations of existing data, propose complementary surveillance resources, and provide examples of promising approaches designed to meet the needs of data-end users. We do not address use of healthrelated surveillance resources for other purposes, such as development of etiological models or evaluation of service access, though these are important challenges and could benefit from the approaches we consider.

\section{SURVEILLANCE DATA NEEDS}

Estimation of healthcare needs and rational allocation of resources are central surveillance data goals. Without timely data, resource allocation falls prey to nonscientific considerations and its attendant inefficiencies or inequities. Surveillance data and modeling of opioid use disorder (OUD) can improve allocation of prevention and treatment resources by stratifying population groups by risk level or identifying groups with special needs. Although surveillance data can suggest biological risk factors, most known risk factors are environmental. Some examples include sociodemographic characteristics [9], geographical location [10], co-occurring health crises [6, 11], and personal history, such as adverse childhood events [12].

Cross-sectional prevalence estimates and correlates become more valuable when they are complemented with trends in substance- and mental health-related outcomes, psychosocial function, and mortality information [13-15]. Trend data can help anticipate needs and redirect resources, as well as help evaluate the effectiveness of interventions, including policy changes and resource re-allocation. Surveillance of emerging hot spots, modeling, and geographic visualization and can be particularly useful in understanding spatial-temporal trends and patterns of opioid use and OUD [16, 17].

\section{DATA END-USERS}

One way to think about surveillance is as a goal in itself. An alternative is to consider the needs of its end-users and organize data collection to meet their needs [18]. Among the most important data end-users are public health systems leaders; public, nonprofit and prepaid healthcare systems, such as health maintenance

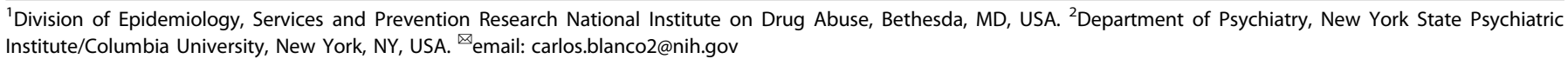

Received: 14 May 2021 Revised: 1 October 2021 Accepted: 6 October 2021

Published online: 29 October 2021 
Table 1. Examples of data, potential end-users, and potential policy actions to improve response to the opioid epidemic.

Data

Geographical distribution of overdoses

Prevalence and pattern of types of opioids in overdoses

Naloxone availability

Polysubstance overdoses

Trends in overdoses

Prevalence and risk factors for substances use and disorders

\section{Potential end-users}

Public Health Departments/Health Systems

Policy Makers Health Systems/ Public Health Departments

Public Health Departments/ Policy Makers

Public Health Departments/ Policy Makers

Public Health Departments/Health Systems/Policy Makers

Public Health Departments/Health Systems

\section{Potential actions}

Deployment of additional resources for emergency care, including naloxone distribution

Prescription drug-monitoring programs and regulations. Regulations to minimize opioid diversion. Improved pain management. Novel approaches to detection of fentanyl lacing and treatment of fentanyl-related overdoses

Distribution of naloxone based on prevalence of overdoses. Revision of regulations governing naloxone dispensation

Modification of clinical overdose reversal protocols. Regulations governing use of funds for substance-specific services

Level and distribution of prevention and treatment resources.

Planning for emergency services and linkages to treatment. Evaluation of existing policies (e.g., legal consequences of drug use, syringe-exchange programs) and funding levels.

Selection of preventive interventions. Allocation and distribution of treatment resources organizations; and other systems, including the criminal justice system, public policy makers, and interested researchers. We summarize some examples below (see also Table 1).

Although there is considerable variation regarding the scope of services they provide, public health systems need data to advocate for resources, and to inform how and where to deploy them [19]. For example, location of naloxone distribution centers can be informed by the geographic distribution of overdoses [20]. Changes in patterns of drug use can also inform resource allocation. Increases in prescription opioid use disorder (POUD), for instance, may require somewhat different approaches than increases in heroin or fentanyl use [21]. While improved training in pain management and reduction of opioid diversion may help curtail POUD, improving detection of fentanyl lacing may be more important to prevention of heroin overdoses.

Public, nonprofit, and prepaid healthcare systems could benefit from timely data to estimate treatment needs, unmet need for emergency services [22, 23], changes in needs [24] and, in the case of population-oriented health systems, plans for prevention $[2,23,25]$.

Policy makers need timely data to make resource allocation decisions. This typically involves balancing competing needs, such as other health-related needs, education, or infrastructure [26, 27]. Yet at present, there are no formal mechanisms to reconcile the needs and interests of surveillance systems and data end-users and modelers. This results in inefficiencies and mismatches between data collection efforts and the informational needs of decision-makers. For example, services funding decisions can be preferentially based on wealth or political influence rather than on the number and distribution of overdoses or OUD prevalence.

Constraints on resources for surveillance combined with multiple data end-users, which may have competing interests, suggest the need for a dialogue between data collecting entities and end-users. Such dialogue could lead to better understanding of end-user needs, increased transparency concerning the goals of data collection, better tailoring of actionable data, and improved dissemination of the results to end-users who can then implement informed policies $[2,18]$. To our knowledge, there are currently no systematic mechanisms to ensure this dialogue. Appointment of representatives of end-users (e.g., associations of public health officials or health system administrators) to advisory boards overseeing publicly funded surveys could help bridge this chasm. Being explicit about survey goals and about data needs of end-users could also improve convergence between the goals and needs of data generators and end-users. For example, more timely information about overdoses could inform distribution of naloxone, while changes in the prevalence or treatment-seeking patterns for OUD could inform location of new clinics or deployment of mobile treatment units.

In some cases, health systems or public health departments collect data but do not have the infrastructure or resources necessary to analyze them beyond simple descriptive reports, as such analyses would be considered research, and thus outside their purview. Challenges exist in creating cultures within health and public health agencies and systems in which data analysis is viewed as central to their mission. Too often this work is viewed as a diversion of resources rather than fundamental to the evaluation of practices and allocation of resources that are key to targeting data collection and evidence-based approaches to public health. One potential solution for localities that are too small to have their own data analytic departments is to join forces with larger nearby jurisdictions, universities, or other data analytic processing centers to share these tasks.

\section{DATA SOURCES}

Existing data sources commonly used for opioid surveillance can be classified into five broad categories [28]: (1) national surveys, (2) electronic health records (EHR) and claims data, (3) mortality records, (4) prescription drug-monitoring data, and (5) contextual and policy data.

National surveys with population-based sampling frames collect information on substance use (including opioids), lifetime or current mental disorders (including substance use disorders), treatment patterns, and sociodemographic characteristics of participants. Examples of these include the National Survey on Drug and Health [29], Monitoring the Future [30], and several surveys and data collection systems funded by the Centers for Disease Control and Prevention (CDC) [31], such as the Behavioral Risk Factor Surveillance System [32] and the National Health Interview Survey [33]. Other national surveys collect information from treatment providers, including clinicians, hospitals, and other treatment facilities. Examples of these include the National Survey of Substance Abuse Treatment Services data [34], the National Ambulatory Medical Care Survey [35], and the Healthcare Cost and Utilization Project [36]. These data systems monitor healthcare use and therefore do not directly reflect the underlying incidence or prevalence of disorders, as many people do not use the healthcare system. There are many factors beyond the disorder and its severity that influence treatment seeking and access to care, including racial/ethnic disparities, socioeconomic status, insurance, and service availability [37].

EHR data are available in real-time and often contain information, such as health behaviors or results of laboratory tests that 
may indicate opioid misuse, but are still challenged by quality issues and limited generalizability due to biases implicit in specific healthcare recording processes [38]. Claims data, which may provide greater coverage than EHR data, tend to have less detailed clinical information and are often expensive to obtain.

Mortality records include the CDC Wonder, the NVSS and the National Death Index (NDI) [39]. CDC WONDER and NVSS support linkages and county-level analyses. The NDI allows for person-level linkages through Social Security Numbers and other personal health information with multiple other databases, including some population-based surveys and treatment utilization data. These linkages have a cost, although the fee can be waived in certain cases for NIH grantees.

Prescription drug-monitoring programs (PDMPs), generally managed by a state authority, collect information on patterns in opioid analgesic prescribing, dispensing, and use. Finally, policy data sources capture information on state opioid policies and thus are generally analyzed and linked using state as the unit of analysis. Contextual data sources are generally used in opioid research to assess state- or county-level factors associated with opioid-related outcomes or to account for time-varying state- or county-level factors that may confound estimation of outcomes in analyses of opioid-related policies.

In addition to these five broad categories, there are a variety of other national, state, or local data sources (e.g., online state opioid dashboards) or proprietary sources such as national poison control centers data, data on surveillance of abuse or diversion (such as the Researched Abuse, Diversion and Addiction-Related Surveillance or RADARS programs) or surveys, such as Ipsos [40] and NORC through its Amerispeak panel [41].

\section{EXISTING CHALLENGES}

While existing surveys provide surveillance information on the opioid epidemic, there are several limitations and challenges.

\section{Data timeliness}

In light of the fast-moving nature of local outbreaks, such as shifting patterns of illicit fentanyl use, more nimble surveillance methods are critically needed. Current data processing and quality control lead to findings becoming available typically more than 6 months and often more than a year after data collection. These delays limit the value of data for planning and resource allocation. Trade-offs persist between speed and accuracy of data. Faster reporting is associated with lower accuracy, but delayed reporting decreases the ability of data to inform service and policy decisions [42].

One solution involves generation of provisional estimates. For example, the CDC generates provisional estimates of overdoses [3], which are revised as more reliable information becomes available. Models that combine data from provisional estimates and alternative indicators and then are calibrated against slower but more reliable data might be able to generate faster yet relatively accurate estimates [43]. Convenience samples, such as internet panels, can also provide rapid information, but approaches to calibrate them against population-based samples and reweight them to obtain generalizable estimates remains underdeveloped. Analysis of internet search terms or analyses of data from social media are other potential surveillance information streams [44].

In some cases, state-level data using distributed research networks can generate data faster than through federal institutions, while addressing concerns about data sharing and privacy [45].

\section{Sample representativeness}

Representativeness of estimates is increasingly constrained by declining rates of survey response [46], non-response bias, and exclusion of important populations at increased risk for substance use and substance use disorders, including especially those in jail or prison, or without stable housing [47]. Exclusion or undercounting of certain populations in the census can distort the survey sampling framework and decrease its representativeness [48]. Furthermore, the accuracy of some data elements, such as cause of death, may be subject to inaccuracies and thereby introduce systematic geographical or racial/ethnic biases in data quality $[49,50]$. Establishment of national standards for data collection and reporting has been suggested as a way to improve data quality and minimize geographical variation and biases [28].

Several surveys are focused on the general population. Although they provide useful information regarding prevalence of several mental disorders and behaviors, they underestimate prevalence of opioid use, OUD, and related outcomes. This is because some groups, such as justice-involved populations or homeless individuals, have higher OUD prevalence [48], but are frequently undersampled. Capture-recapture epidemiological methods can improve estimates of prevalence of OUD by leveraging how often an individual with OUD is identified in one or more relevant databases (e.g., healthcare or justice systems) [51]. In the future, it will be important to develop, and implement approaches that capture information from high-risk or hard to reach populations [52], to obtain more accurate overall prevalence estimates and ensure appropriate service planning for these populations. A complementary strategy involves triangulation, such as reweighting convenience samples using reference populations $[53,54]$ or combining information from different sources, e.g., population-based surveys with treatment data or with data from digital surveillance methods (Twitter, Google, and others) [44].

\section{Populations variability}

Geographic or sociodemographic variations in prevalence of opioid use, OUD, and overdoses pose challenges regarding assessment and service planning needs. Jurisdictions often seek local data to inform their decision-making. Except for highly prevalent disorders, however, local data can be sparse and lead to unstable estimates. There is a need to develop and implement methods that combine different data sources, such as Bayesian approaches, that could use national data as priors and incorporate local data to better approximate the prevalence in specific jurisdictions. Combining data from general population surveys with data from the healthcare or justice systems can also generate more stable and precise local estimates.

Simulation models, which have been widely used to estimate the spread and transmission of the COVID-19 pandemic and forecast its population incidence after the implementation of interventions $[55,56]$, can also be useful for similar purposes in the opioid epidemic $[57,58]$. An important area of research will be development of methods to generalize results of simulations and project rates of the epidemic and effects of the interventions in populations other than those used to generate the initial models. More generally, discussions between developers of the simulation models and end-users may be necessary to optimize trade-offs between dedicating resources to collect ever more local data versus funding other community needs, such as increased availability of treatment or prevention services [18].

\section{Limited flexibility}

Most existing data collection systems lack the necessary flexibility to adapt to shifts in the environment, including during pandemics or other disasters. Infrastructure or procedures that increase flexibility and responsiveness to changing conditions without substantially increasing the cost may help data collection efforts to become more relevant to changing environments.

\section{Database linkage}

Data linkage is critical to achieve most goals of interest to endusers. For example, linking individual overdose rescue data with 
medical records could provide new insights about risk factors for overdose, as well as more systematic information on the consequences of nonfatal overdoses. Linkage to the location of emergency departments and treatment facilities could inform deployment of additional resources, while combination of EHR, claims data and population-based surveys could shed light on which types of individuals are seeking treatment and which populations may face barriers to treatment. PDMPs could help identify the proportion of individuals with overdoses receive opioids from their clinicians versus illegal markets, whereas contextual and policy data may help determine whether changes in policies are effective in decreasing overdoses.

Despite these potential advantages, the existing infrastructure makes linking databases time consuming and resource-intensive, especially when it involves crossing data systems, raising issues of interoperability [59]. Because linkages are expensive, data collectors and end-users need to establish priorities regarding which data linkages are most likely to yield novel insights and inform critical public health decisions. For example, the benefits of linking individual overdose data with a variety of databases need to be compared with others, such as collecting better data on unreported naloxone rescues (which could help identify population at risk), the overlap between suicide and overdose, the complexity of overdose death with multiple substances detected or the number of individuals who die alone of an overdose.

Linkages can also pose threats to confidentiality that must be carefully managed. Achieving the right balance between data availability and privacy protection has become more challenging as increased computational power and availability of data derived from the Internet has made it easier to reidentify participants. Ongoing efforts including blockchain-based approaches [60] seek to develop privacy preserving record linkages that do not require sharing personally-identifying information among disparate organizations. Yet these technologies are still years away [61]. In other cases, such as studying the effects of policies on populations, it may not be necessary to link databases at the individual level, thereby reducing cost and eliminating concerns over re-identification. Linking at the supra-individual level is generally less resource-intensive, making these linked datasets more readily available to a broad range of researchers and other end-users. Developing infrastructure and methods to facilitate linkages at different levels could leverage use of existing data that are currently suboptimally used.

\section{Longitudinal data}

All of these challenges become more difficult in longitudinal surveys and cohorts followed within EHR and claims data. For example, representativeness is further threatened by loss to follow up from non-response in surveys or departures by patients from health systems in administrative data, which adds to cost and complicates design and analysis. As compared to cross-sectional data, longitudinal data also require more complex and timely data processing and analysis. Longitudinal information can also increase risks of re-identification, requiring additional levels of privacy protection.

\section{PROMISING EXAMPLES AND FUTURE DIRECTIONS}

There are some promising developments in infrastructure and methods. For example, the Washington/Baltimore Drug Intensity Traffic Area (W/B HIDTA) has developed the Overdose Detection Mapping Application Program (ODMAP) [62]. ODMAP provides near real-time suspected overdose surveillance data across jurisdictions by linking first responders and relevant record management systems (e.g., EMS, law enforcement and healthcare data) to a mapping tool to track overdoses to stimulate real-time response and strategic analysis across jurisdictions. ODMAP is currently only available to government (state, local, federal, or tribal) agencies serving the interests of public safety and health. However, government agencies may choose to provide access to ODMAP to nonprofit agencies, by registering individuals under the government agency. ODMAP offers a promising example of how different data streams can be integrated for better analyses and faster public health response.

Automatic data extraction from electronic medical records (EHRs) or other databases, sometimes combined with natural language processing methodologies, can broaden information sources for opioid surveillance. Distributed data networks such as those used in Medicaid Outcomes Distributed Research Network [45] or the FDA sentinel system [63] allow the combination of EHR data from multiple health systems, while preserving the privacy of the individuals in those systems.

A series of projects have also sought to better integrate data collection, statistical modeling, and public health interventions. For example, a NIDA-funded study is developing predictive analytics models in Rhode Island to forecast future overdose mortality at the neighborhood-level, using publicly available information and data from a multicomponent overdose surveillance system [64]. The results might better inform resource allocation to communities in greatest need of prevention, treatment, recovery, and overdose rescue services.

As part of another initiative, the Massachusetts Department of Public Health has linked 28 government databases to establish an integrated data warehouse to inform the state's response to the opioid epidemic, including clinical guidance and policy decisions [19]. This initiative uses project-specific identification numbers to minimize risk of re-identification. Partnerships with researchers have led to several influential publications [51, 65]. This model could also be replicated in other jurisdictions.

Other developments rely on alternative or complementary sources of information. For example, wastewater [66] or drug testing samples [67] are being studied as means of estimating population changes in opioid use. Cell phones and virtual technologies also offer alternative means of data collection. Use of wearables that monitor patients' activities without physical contact (e.g., sleep and heart rate variability) and mailing of biological specimens can also be used in selected samples for more intensive data collection.

\section{CONCLUSION}

Given fiscal constraints on federal and state government budgets and urgent competing public health needs posed by COVID-19, it remains unclear how public health authorities and private healthcare systems will respond to surveillance challenges needed to more effectively combat the opioid epidemic. Traditional data collection systems are slow, labor-intensive, expensive, and have been insufficient to provide the information needed to control the epidemic. There is a pressing need to develop and implement more efficient and nimble approaches. Because improved surveillance would benefit multiple public and private constituencies and end-users, a public debate will likely ensue over how to finance development and implementation of these activities.

Accelerating data reporting will require increasing the capacity of states and other relevant jurisdictions to collect, process, and transmit data, while maintaining quality standards. Because of financial constraints, efforts to improve interoperability and link databases will require difficult decisions about prioritizing linkages. Although focused on opioid surveillance, many of these themes apply, with small variations, to the surveillance of cocaine, methamphetamine, and other substances.

Despite these challenges, there is increased awareness of the need to use data in real-time to inform policy and public health decisions. There is growing interest in collaborations and keen interest in developing new data collection and modeling methods. These trends together with progress in public health learning 
systems of care offer new possibilities to advance science, combat the opioid epidemic, and save lives.

\section{REFERENCES}

1. President's Commission on Combating Drug Addiction and the Opioid Crisis. 2017. Final_Report_Draft_11-15-2017.pdf (archives.gov). Accessed 25 June 2021.

2. Blanco C, Wiley TRA, Lloyd JJ, Lopez MF, Volkow ND. America's opioid crisis: the need for an integrated public health approach. Trans Psychiatry. 2020;10:167.

3. National Vistal Statistics System. Vital Statistics Rapid Release-Provisional Drug Overdose Data. https://www.cdc.gov/nchs/nvss/vsrr/drug-overdose-data.htm. Accessed 25 June 2021

4. Volkow ND, Blanco C. The changing opioid crisis: development, challenges and opportunities. Mol Psychiatry. 2021;26:218-33.

5. Jewell NP, Lewnard JA, Jewell BL. Predictive mathematical models of the COVID-19 pandemic: underlying principles and value of projections. JAMA. 2020;323:1893-4.

6. Blanco C, Compton WM, Volkow ND. Opportunities for research on the treatment of substance use disorders in the context of COVID-19. JAMA Psychiatry. 2021;78:357-8.

7. Peters PJ, Pontones P, Hoover KW, Patel MR, Galang RR, Shields J, et al. HIV infection linked to injection use of oxymorphone in Indiana, 2014-2015. NEJM. 2016;375:229-39.

8. Han E, Tan MMJ, Turk E, Sridhar D, Leung GM, Shibuya K, et al. Lessons learnt from easing COVID-19 restrictions: an analysis of countries and regions in Asia Pacific and Europe. Lancet. 2020;396:1525-34. https://doi.org/10.1016/S0140-6736(20) 32007-9.

9. Han B, Compton WM, Blanco C, Crane E, Lee J, Jones CM. Prescription opioid use, misuse, and use disorders in US adults: 2015 National Survey on Drug Use and Health. Ann Int Med. 2017;167:293-301.

10. Scholl L, Seth P, Kariisa M, Wilson N, Baldwin G. Drug and opioid-involved overdose deaths-United States, 2013-2017. MMWR. 2019;67:1419-27.

11. Volkow ND. Collision of the COVID-19 and addiction epidemics. Ann Int Med. 2020;173:61-2.

12. Merrick MT, Ford DC, Haegerich TM, Simon T. Adverse childhood experiences increase risk for prescription opioid misuse. J Prim Prev. 2020;41:139-52.

13. Blanco C, Alderson D, Ogburn E, Grant BF, Nunes EV, Hatzenbuehler ML, et al. Changes in the prevalence of non-medical prescription drug use and drug use disorders in the United States: 1991-1992 and 2001-2002. Drug Alcohol Depend. 2007;90:252-60.

14. Saha TD, Kerridge BT, Goldstein RB, Chou SP, Zhang H, Jung J, et al. Nonmedical prescription opioid use and DSM-5 nonmedical prescription opioid use disorder in the United States. J Clin Psychiatry. 2016;77:772-80.

15. Olfson M, Rossen LM, Wall MM, Houry D, Blanco C. Trends in intentional and unintentional opioid overdose deaths in the United States, 2000-2017. JAMA. 2019;322:2340-2.

16. Langabeer JR, Gourishankar A, Chambers KA, Giri S, Madu R, Champagne-Langabeer T. Disparities between US opioid overdose deaths and treatment capacity: a geospatial and descriptive analysis. J Addict Med. 2019;13:476-82.

17. Basak A, Cadena J, Marathe A, Vullikanti A. Detection of spatiotemporal prescription opioid hot spots with network scan statistics: multistate analysis. JMIR Public Health Surveill. 2019;5:e12110.

18. Blanco C, Lopez MF, Goldstein AB. User-centered psychiatric epidemiology. JAMA Psychiatry. 2020;77:992-3.

19. Bharel M, Bernson D, Averbach A. Using data to guide action in response to the public health crisis of opioid overdoses. NEJM Catalyst Inn Care Delivery. 2020;05. https://doi.org/10.1056/CAT.19.1118.

20. Belz D, Lieb J, Rea T, Eisenberg MS. Naloxone use in a tiered-response emergency medical services system. Prehosp Emerg Care. 2006;10:468-71.

21. Shearer RD, Howell BA, Bart G, Winkelman TN. Substance use patterns and health profiles among US adults who use opioids, methamphetamine, or both, 2015-2018. Drug Alcohol Depend. 2020;214:108162.

22. Campbell $\mathrm{Cl}$, Weisner $\mathrm{C}$, Binswanger IA, Lapham GT, Ahmedani BK, Yarborough $\mathrm{BJH}$, et al. Predictors of healthcare effectiveness data and information set (HEDIS) treatment initiation and engagement among patients with opioid use disorder across 7 health systems. Subst Abus. 2019;40:328-34.

23. Campbell Cl, Bahorik AL, VanVeldhuisen P, Weisner C, Rubinstein AL, Ray GT. Use of a prescription opioid registry to examine opioid misuse and overdose in an integrated health system. Prev Med. 2018;110:31-7.

24. Slavova S, Rock P, Bush HM, Quesinberry D, Walsh SL. Signal of increased opioid overdose during COVID-19 from emergency medical services data. Drug Alcohol Depend. 2020;214:108176.

25. Blanco C, Volkow ND. Management of opioid use disorder in the USA: present status and future directions. Lancet. 2019:393:1760-72.
26. Miller G, Rhyan C, Beaudin-Seiler B, Hughes-Cromwick P. A framework for measuring low-value care. Value Health. 2018;21:375-9.

27. Angelis A, Kanavos P, Phillips LD. ICER Value Framework 2020 Update: recommendations on the aggregation of benefits and contextual considerations. Value Health. 2020;23:1040-8.

28. Smart R, Kase CA, Meyer A, Stein BD. Data Sources and Data-Linking Strategies to Support Research to Address the Opioid Crisis. Final Report. U. S. Department of Health and Human Services. Assistant Secretary for Planning and Evaluation. Office of Health Policy. This report was prepared under contract \# HHSP23320095649WC. The task order number for the current Time \& Materials umbrella contract is: HHSP23337038T between HHS's ASPE/HP and the RAND Corporation. 2018.

29. Substance Abuse and Mental Heatlth Administration. National Survey on Drug Use and Health. https://www.samhsa.gov/data/release/2019-national-surveydrug-use-and-health-nsduh-releases. Accessed 29 April 2021.

30. Monitoring The Future. http://www.monitoringthefuture.org/. Accessed 29 April 2021.

31. Centers for Disease Control and Prevention. Opioid Data Analysis and Resources. https://www.cdc.gov/drugoverdose/data/analysis.html. Accessed 24 Mar 2021.

32. Centers for Disease Control and Prevention. Behavioral Risk Surveillance System. https://www.cdc.gov/brfss/index.html. Accessed 29 April 2021.

33. Centers for Disease Control and Prevention. National Health Interview Survey https://www.cdc.gov/nchs/nhis/index.htm. Accessed 29 April 2021.

34. Substance Abuse and Mental Health Administration. National Survey of Substance Abuse Treatment Services (N-SSATS). https://wwwdasis.samhsa.gov/ dasis2/nssats.htm. Accessed 29 April 2021.

35. Centers for Disease Control and Prevention. National Center for Health Statistics. About the Ambulatory Health Care Surveys. https://www.cdc.gov/nchs/ahcd/ about_ahcd.htm. Accessed 29 April 2021.

36. Agency for Health Research and Quality. Healthcare Cost and Utilization Project (HCUP). https://www.ahrq.gov/data/hcup/index.html. Accessed 29 April 2021.

37. Blanco C, Iza M, Schwartz RP, Rafful C, Wang S, Olfson M. Probability and predictors of treatment-seeking for prescription opioid use disorders: a national study. Drug Alcohol Depend. 2013;131:143-8.

38. Ta $C N$, Weng $C$. Detecting systemic data quality issues in electronic health records. Stud Health Technol Inform. 2019;264:383-7.

39. Centers for Disease Control and Prevention. National Death Index. https://www. cdc.gov/nchs/ndi/index.htm. Accessed 29 April 2021.

40. Ipsos | Global Market Research and Public Opinion Specialist. Available from: https://www.ipsos.com/en. Accessed 29 April 2021.

41. AmeriSpeak. https://www.amerispeak.org/. Accessed 29 April 2021.

42. Spencer MR, Ahmad F Timeliness of death certificate data for mortality surveillance and provisional estimates. National Center for Health Statistics. 2017 https://www.cdc.gov/nchs/data/vsrr/report001.pdf. Accessed 29 April 2021.

43. Rossen LM, Branum AM, Ahmad FB, Sutton PD, Anderson RN. Notes from the Field: Update on Excess Deaths Associated with the COVID-19 Pandemic-United States, January 26, 2020-February 27, 2021. MMWR Morb Mortal Wkly Rep. 2021;70:570-1. https://doi.org/10.15585/mmwr.mm7015a4.

44. Kalyanam J, Mackey TK. A review of digital surveillance methods and approaches to combat prescription drug abuse. Curr Addict Rep. 2017;4:397-409.

45. Lo-Ciganic $\mathrm{W}-\mathrm{H}$, Huang $\mathrm{J}$, Zhang $\mathrm{HH}$, Weiss $\mathrm{JC}, \mathrm{Wu} \mathrm{Y}, \mathrm{Kwoh} \mathrm{CK}$, et al Evaluation of machine-learning algorithms for predicting opioid overdose risk among medicare beneficiaries with opioid prescriptions. JAMA Net Open. 2019;2: e190968.

46. National Research Council. Nonresponse in social science surveys: a research agenda. Plewes TJ, Tourangeau R, editors. Panel on a Research Agenda for the Future of Social Science Data Collection, Committee on National Statistics, Division of Behavioral and Social Sciences and Education. Washington, DC: The National Academies Press; 2013.

47. Compton WM, Dawson D, Duffy SQ, Grant BF. The effect of inmate populations on estimates of DSM-IV alcohol and drug use disorders in the United States. Am J Psychiatry. 2010;167:473-4.

48. Centers for Disease Control and Prevention. National Health Interview Survey. https://www.cdc.gov/nchs/nhis/index.htm. Accessed 29 April 2021.

49. Tote KM, Bradley $H$, Martin EG, Yucel R, Rosenberg ES. Factors associated with incomplete toxicology reporting in drug overdose deaths, 2010-2016. Ann Epidemiol. 2019;38:65-9.

50. Davis G. National Association of Medical Examiners and American College of Medical Toxicology Expert Panel on Evaluating and Reporting Opioid Deaths. National Association of Medical Examiners position paper: recommendations for the investigation, diagnosis, and certification of deaths related to opioid drugs. Acad Forensic Pathol. 2013;3:77-83.

51. Barocas JA, White LF, Wang J, Walley AY, LaRochelle MR, Bernson D, et al Estimated prevalence of opioid use disorder in Massachusetts, 2011-2015: a capture-recapture analysis. Am J Public Health. 2018;108:1675-81. 
52. National Institute of Justice. NIJ's Drugs and Crime Research: Arrestee Drug Abuse Monitoring Programs. https://www.nij.gov/topics/drugs/markets/adam/pages/ welcome.aspx. Accessed 26 Mar 2021.

53. DuGoff EH, Schuler M, Stuart EA. Generalizing observational study results: applying propensity score methods to complex surveys. Health Ser Res. 2014;49:284-303.

54. Blanco C, Campbell AN, Wall MM, Olfson M, Wang S, Nunes EV. Toward national estimates of effectiveness of treatment for substance use. J Clin Psychiatry. 2017;78:64-70.

55. Adam D. Special report: The simulations driving the world's response to COVID19. Nature. 2020;580:316-9.

56. Hoertel N, Blachier M, Blanco C, Olfson M, Massetti M, Rico MS, et al. A stochastic agent-based model of the SARS-CoV-2 epidemic in France. Nat Med. 2020;26: 1417-21.

57. Linas BP, Savinkina A, Madushani R, Wang J, Yazdi GE, Chatterjee A, et al. Projected estimates of opioid mortality after community-level interventions. JAMA Net Open. 2021;4:e2037259.

58. Volkow ND, Blanco C. Interventions to address the opioid crisis-modeling predictions and consequences of inaction. JAMA Net Open. 2021;4:e2037385.

59. Reis ZSN, Maia TA, Marcolino MS, Becerra-Posada F, Novillo-Ortiz D, Ribeiro ALP. Is there evidence of cost benefits of electronic medical records, standards, or interoperability in hospital information systems? Overview of systematic reviews. JMIR Med Inform. 2017;5:e7400.

60. Cernian A, Tiganoaia B, Sacala I, Pavel A, Iftemi A. PatientDataChain: a blockchainbased approach to integrate personal health records. Sensors. 2020;20:6538.

61. Dullabh P, Dhopeshwarkar R, Heaney-Huls K, Hovey L, Desai P, Lama S, et al. Building the Data Capacity for Patient-Centered Outcomes Research: The 2020 Annual Report. Prepared under Contract No. HHSP233201600020I. The task order number for the current Cost Plus Fixed Fee umbrella contract is: HHSP23337001T between the Department of Health and Human Services' Office of the Assistant Secretary for Planning and Evaluation Office of Health Policy and NORC at the University of Chicago.

62. Overdose Detection Mapping Application Program. http://www.odmap.org/ \#home. Accessed 29 April 2021.

63. Platt R, Brown JS, Robb M, McClellan M, Ball R, Nguyen MD, et al. The FDA sentinel initiative-an evolving national resource. N Engl J Med. 2018;379:2091-3.

64. NIH Research Portfolio Online Reporting Tools Expenditures and Results (NIH RePORTER) https://reporter.nih.gov/search/yHy7ON7fcU6oxESHMdalng/projectdetails/10220922. Accessed 4 Nov 2020.
65. Kimmel SD, Walley AY, Li Y, Linas BP, Lodi S, Bernson D, et al. Association of treatment with medications for opioid use disorder with mortality after hospitalization for injection drug use-associated infective endocarditis. JAMA Net Open. 2020;3:e2016228.

66. Been F, Benaglia L, Lucia S, Gervasoni J-P, Esseiva P, Delémont O. Data triangulation in the context of opioids monitoring via wastewater analyses. Drug Alcohol Depend. 2015;151:203-10.

67. Stanton JD, Whitley P, LaRue L, Bundy WL, Dawson E, Huskey A. Fentanyl analog positivity among near-real-time urine drug test results in patients seeking health care. Drug Alcohol Depend. 2020;217:108264.

\section{ACKNOWLEDGEMENTS}

The views and opinions expressed in this report are those of the authors and should not be construed to represent the views of any of the National Institute on Drug Abuse, the National Institutes of Health or any US government agency.

\section{AUTHOR CONTRIBUTIONS}

Conception and design, acquisition of data, or analysis and interpretation of data: $C B$, MMW, and MO. Drafting the article: CB. Revising it critically for important intellectual content: MMW, MO. Final approval of the version to be published: all authors.

\section{COMPETING INTERESTS}

The authors declare no competing interests.

\section{ADDITIONAL INFORMATION}

Correspondence and requests for materials should be addressed to Carlos Blanco.

Reprints and permission information is available at http://www.nature.com/ reprints

Publisher's note Springer Nature remains neutral with regard to jurisdictional claims in published maps and institutional affiliations. 